\title{
Potential Development of a Mobile Application for Gout Self-Management: What Support Do Patients Need?
}

\author{
Yao Yin ${ }^{\prime}$ \\ Huan Wang ${ }^{2}$ \\ Chao-Feng Fan' \\ Hong Chen ${ }^{3}$ \\ 'Department of Neurology/Evidence- \\ based Nursing Center, West China \\ Hospital, Sichuan University, Chengdu, \\ Sichuan Province, People's Republic of \\ China; ${ }^{2}$ Department of Nursing, The \\ Third Hospital of Mianyang, Mianyang, \\ Sichuan Province, People's Republic of \\ China; ${ }^{3}$ West China School of Nursing \\ and Department of Nursing, West China \\ Hospital, Sichuan University, Chengdu, \\ Sichuan Province, People's Republic of \\ China
}

Purpose: With the increasing prevalence and chronic course of gout, the use of mobile health (mHealth) applications (APPs) for gout self-management is attracting considerable research attention as an effective approach to improving health outcomes. However, there is limited knowledge regarding the self-management support that a mHealth APP for gout should provide to patients according to their needs. The aim of this study was to interview patients with gout and identify their needs with respect to gout self-management support.

Patients and Methods: We conducted qualitative research using a purposive sampling method from January 2019 to August 2019. Individual face-to-face semi-structured interviews were conducted with patients recruited from West China Hospital in Sichuan, China. The qualitative data were transcribed verbatim and analyzed by thematic analysis.

Results: Ten male patients with gout were included in the final analysis. Instrumental, psychological, and relational support emerged as themes describing the needs of patients for gout self-management support. The instrumental support included health education, hospital registration, setting reminders, and shopping. Psychological support referred to helping patients mitigate various negative emotions. Relational support included interactions with healthcare providers and fellow patients.

Conclusion: The present findings indicate that healthcare providers or companies should develop mHealth APPs for gout self-management based on the needs of patients.

Keywords: gout, self-management, mobile applications, China

\section{Introduction}

Gout is the most common type of inflammatory arthritis. ${ }^{1}$ Although it is more prevalent among men aged $>40$ years (accounting for $90.0-95.0 \%$ of cases), it also occurs in women after menopause. ${ }^{2}$ A meta-analysis reported that the pooled prevalence of gout in mainland China was $1.1 \%$ (95\% confidence interval: 0.8 $1.5 \%$ ), which is lower than that observed in Taiwan Province $(6.2 \%)^{3,4}$ The incidence of gout also shows an increasing trend in China. ${ }^{4}$ Most patients require self-management with lifelong urate-lowering therapy (ULT), self-medication following the occurrence of flare-warning symptoms, management of chronic comorbidities, and lifestyle adjustment. ${ }^{2,5-7}$ However, evidence showed that $77.9 \%$ of patients with gout had poor self-management, with only $15.4 \%$ achieving clinical remission. ${ }^{8,9}$

With the increasing use of electronic devices, mobile health (mHealth) applications (APPs) are attracting considerable research attention as an effective approach
Correspondence: Hong Chen

West China School of Nursing and

Department of Nursing, West China

Hospital, Sichuan University, No. 37,

Guoxuexiang, Wuhou District, Chengdu,

Sichuan Province, 61004I, People's

Republic of China

$\mathrm{Tel}+8618980601733$

Email1366109878@qq.com 
to promoting self-management for patients with chronic diseases. $^{10,11}$ The NEWZOO 2020 Global Mobile Market Report $^{12}$ reported that the number of smartphone users reached 3.6 billion in 2020. China accounted for more than a quarter of these users (nearly 850 million). By 2023 , there will be 4.1 billion smartphone users globally, increasing between 2018 and 2023 at a compound annual growth rate of $+6.2 \%$. Furthermore, the number of downloads of mHealth APPs nearly doubled between 2013 and 2016. According to Research2Guidance, 2.6 billion users were expected to download mHealth APPs using mobile phones or tablets by 2020 . A survey found that $86.1 \%$ of patients with gout were willing to use mHealth APPs for gout self-management. ${ }^{13}$ Empirical evidence reported that using disease-related APPs could significantly improve disease-related knowledge, diet and medication adherence, and perceived social support, as well as reduce anxiety and depression, and ultimately improve self-management and health outcomes. ${ }^{14-16}$ In 2019, we reported on existing APPs related to gout in China. ${ }^{17}$ However, to the best of our knowledge, there is no related study investigating the development of gout-related APPs in China. According to the recommendation of the European Alliance of Associations for Rheumatology, mHealth APPs should be relevant and tailored to the individual needs of patients with rheumatic and musculoskeletal diseases. ${ }^{18}$ User involvement has been widely used in the design and development of such APPs. This approach plays a crucial role in achieving user engagement and improves the likelihood of producing an effective APP. ${ }^{19}$ Thus, in this study, we recruited patients with gout to investigate their needs regarding self-management support with the aim of designing and developing a mobile APP for gout selfmanagement.

\section{Patients and Methods \\ Design}

This study used a qualitative design, wherein individual, face-to-face, semi-structured interviews with gout patients were conducted.

\section{Patients}

Patients were recruited from the rheumatology clinic or ward in West China Hospital in Sichuan, China. The qualitative data were collected from January 2019 to August 2019. Patients with different ages, occupations, and disease stages were selected through a purposive sampling method. The inclusion criteria were: 1) diagnosis of gout for $\geq 6$ months, according to the American College of Rheumatology/European League Against Rheumatism Gout Classification Criteria; ${ }^{20}$ 2) age $\geq 18$ years; 3 ) absence of mental illness and cognitive impairment; and 4) voluntary participation. The sample size was determined by data saturation. Finally, 10 patients were included in this study.

\section{Data Collection}

The interview guide was developed based on a literature review, searching for mobile APPs in APP stores and a pilot study. The interview guide is provided as a supplementary file. One researcher (H.C.), an experienced clinical and qualitative study expert, ensured sufficient coverage and appropriateness of the interview guide. H.C. was also involved in the interview process to ensure research quality. Prior to this study, Y.Y and H.W. received formal training on conducting interviews. All interviews were conducted by Y.Y. to ensure a homogeneous data collection process. Prior to the interview, the main researcher (Y.Y.) informed patients of the research aim, procedures, potential risks and benefits, and confidentiality. Y. Y. had no current healthcare provider relationship with any of the patients. During the data collection process, patients agreed to have their interviews audio-recorded. Handwritten notes were also obtained to record their non-verbal behavior as additional materials. The duration of each interview was 25 $40 \mathrm{~min}$.

\section{Data Analysis}

Within 24h after each interview, two researchers (Y.Y. and H.W.) listened to the recorded interviews and transcribed the content into Chinese verbatim. Subsequently, the transcripts were double-checked and imported to NVivo11.0 (QSR International, Melbourne, Australia). Data analysis followed the Colaizzi's seven steps for the coding and analysis of the transcripts. ${ }^{21}$ Firstly, two researchers (Y.Y. and H.W.) independently read and coded the data. Secondly, codes were grouped into sub-themes and, next, into themes. The two researchers discussed the appropriateness and meaning of the themes prior to reaching an agreement. Finally, three themes were derived from the needs of patients for selfmanagement support, which could be utilized for developing a mHealth APP for gout self-management.

\section{Ethical Considerations}

This study was approved by the West China Hospital Medical Ethics Committee (No. 397[2018]). Written informed consent was provided by all patients. This 
study was conducted in accordance with the tenets of the Declaration of Helsinki.

\section{Results}

\section{Characteristics of the Patients with Gout}

All patients were male, with a median age of 46 years (range: 29-60 years). The median duration of gout was 8 years (range: 1-17 years). Three patients had comorbidities and four had tophi. The characteristics of the patients are shown in Table 1.

\section{Usage and Preference for mHealth APPs}

All patients responded that they had never used a mHealth APP for gout self-management or heard about a goutrelated mHealth APP. One patient also voiced his concerns that he could not fully trust such APPs. Nevertheless, the results showed that all patients were willing to use a mHealth APP to cope with gout.

\section{Self-Management Support Needs of Patients with Gout}

\section{Theme I: Instrumental Support}

\section{Health Education}

Nine health education needs emerged from the qualitative data: basic disease-related knowledge; up-to-date medical news and information related to gout; dietary advice; lifestyle advice; associated comorbidities; prevention and management of an acute attack; treatment of tophi; ULT; and effects and side effects of medications for gout.

\section{Basic Disease-Related Knowledge}

G4: My level of serum uric acid (SUA) is not too high, which is up to $500 \mu \mathrm{mol} / \mathrm{L}$. I know that the SUA of my friends ranges from 800 to $900 \mu \mathrm{mol} / \mathrm{L}$. However, they did not get pain. So, what's the relationship between SUA and gout?

G6: I mainly want to know how many stages of gout disease there are, the symptoms of each stage, and the corresponding effects on me. I do not know if I am in the preliminary stage or the more severe stage.

G7: None of my family members ever had gout. Doctors have never explained clearly why I have gout.

Up-to-Date Medical News and Information Related to Gout

G3: What I am most concerned about is the drug for ULT. Domestically, there is no drug with obvious therapeutic effects for gout. Maybe some drugs are marketed abroad with good therapeutic effect, which are not available on the Internet.

G8: I am now aware of the basic knowledge of medication and diet. I would like to be informed about the latest medication making gout curable.

\section{Dietary Advice}

G3: I really want to know what type of food is forbidden, every doctor that I met has different opinions.

G7: I need information about the diet for gout. What I can and cannot eat? And how much can I eat? I am even scared before eating.

\section{Lifestyle Advice}

G3: I am confused about some advice, such as regular exercise. I do not know if it's good for my health. Some people say that exercise can be hard on the joints, while others say that it is beneficial. Is it suitable for me? Everyone is not sure about this.

G7: I know that gout affects my joints. So, will it be possible for me to continue doing strenuous

Table I Characteristics of Patients

\begin{tabular}{|l|l|l|l|l|l|l|l|l|}
\hline No. & Age & BMI & Marital Status & Education & Occupation & Duration of Disease, Years & Comorbidities & Tophi \\
\hline GI & 49 & 25.3 & Married & College & Civil servant $^{\mathrm{a}}$ & 17 & No & No \\
G2 & 44 & 27.8 & Married & Junior high school & Civil servant $^{\mathrm{a}}$ & 10 & Renal impairment & Yes \\
G3 & 40 & 23.7 & Married & College & Driver & 8 & No & Yes \\
G4 & 30 & 21.3 & Single & College & Police officer & 8 & No & No \\
G5 & 49 & 25.4 & Married & Primary school & Peasant & 10 & No & No \\
G6 & 52 & 23.1 & Married & Junior high school & Driver & 4 & No \\
G7 & 29 & 26.0 & Married & College & Civil servant & 1 & No \\
G8 & 48 & 24.5 & Married & College & Self-employed & 8 & Hyperlipemia & Yes \\
G9 & 60 & 26.7 & Married & Junior high school & Retiree & 9 & Diabetes \\
GI0 & 32 & 26.6 & Married & High school & White collar & & 6 & Yes \\
\hline
\end{tabular}

Notes: ${ }^{a}$ Civil servant: person who is employed in the public sector by a government department or agency. ${ }^{b}$ White collar: clerical worker. Abbreviations: BMI, body mass index. 
exercises like basketball? I really like playing basketball.

G10: I bought a bicycle. And I cycle to my driving school for $2 \mathrm{~h}$ every day. I get wet all over each time when I come back home.

\section{Associated Comorbidities}

G8: I did not care about it before. Because I thought it only had an impact on the SUA. I did not expect that it would affect my kidney function.

G9: I did not know gout would cause kidney damage. Now I have kidney stones, which makes me urinate a lot at night. I cannot sleep well.

\section{How to Prevent and Manage an Acute Attack}

G3: I hope that it will include a guide to prevent pain. And if it happens, how to treat and deal with the pain.

G6: Nobody can explain clearly how to prevent it. It means that as soon as the symptoms appear, I need to take medication for that.

\section{Treatment of Tophi}

G3: I earn a living as a driving coach. But my foot has stones now, it is really inconvenient. How can I cure it?

G4: I want to do surgery to remove the stone from my elbow, but the doctor refused. I thought it could be cured by surgery.

Ult

G7: I did not know that I need to take medication for a long time. The doctor only prescribes medication for me, without telling me that I need to take it for a long time. So, once the pain is relieved, of course, I stop taking medication.

G9: I did not know there are drugs for decreasing uric acid. I would have gotten the treatment if I knew. I had been taking medication for hypertension for 17 years. I thought high uric acid was caused by food. I came to this hospital, then I knew I had to take medications for lowering uric acid.

\section{Effects and Side Effects of Gout Medications}

G1: Information about the side effects of medications is important for me. Every medicine has its side effects. That is why I stopped taking medications, although my level of SUA is well controlled. The side effects of each medicine should be summarized in a table, making it concise. That would be helpful for us.

G7: The side effects of medications are the most concerning for me. I am afraid that they will affect other functions of my body.
G9: I do not know much about the effects of these medications. My friend is a doctor. He told me to take it for gout. I trust him, so I did not try understanding its effects.

G10: The medication that I usually take can also lower my SUA. It is colchicine, I search its effects on the Internet.

\section{Hospital Registration}

Hospital registration offers patients the opportunity to arrange an appointment with the doctor online without having to visit the hospital, thereby saving time.

G4: Sometimes the pain lasted for more than half a month. The medications did not work. So, I need to go to hospital. It will be convenient if I can complete hospital registration online.

\section{Setting Reminders}

Setting reminders helped patients to adhere to their medication and exercise schedules.

G2: I always take the medications for anti-inflammation when some warning signs appear. I also take medications for ULT regularly, but sometimes I forget.

G3: Doctors advise us to do regular exercise. I hope there is an alarm to remind me to keep doing it.

\section{Shopping}

It is necessary for patients to purchase some products/ medications for the self-treatment of gout flares, such as ice bag, and nonsteroidal anti-inflammatory drugs. The mHealth APP could offer this option.

G1: I used to get treatment by traditional Chinese medicine in this hospital. It really showed good effects. But it is far away from where I live. Sometimes, I ask my friend to buy this and send it to me by express delivery.

G2: I want to know where I can buy the medications with better effects. I know I need to be adherent to dietary and exercise advice, but I cannot make it.

\section{Theme 2: Psychosocial Support}

Negative emotions in patients are developed due to the pain, economic burden, fear of medication-related side effects, reduced social activities, change in employment, and disharmony of family relationships caused by gout.

G2: I need to take medications regardless of the occurrence of an attack. This caused an economic burden on my family. When I feel pain, I cannot do a lot of things. For example, I would not do this job if not for gout. The salary is far less than my 
previous job. And my wife is not satisfied with this job, we quarrel sometimes ...

G5: The pain is really annoying. Being a man, I can only stay at home, I cannot work and make money. I feel a little frustrated, but just a few times.

G8: Sometimes I am a little upset, I need to stay in bed at home. I cannot eat what I like, I was told to control my diet. In fact, I have a good appetite, I like traveling to where I can eat local delicacies. But there is a lot of food that I cannot eat now. It is really a pity for me.

G10: Actually, it has a little impact on my work. But I have a heavy mental load because of frequent gout flares. And I found information about the severe side effects of colchicine. I am wondering if I will get other problems because of the medications. I am really anxious.

\section{Theme 3: Relational Support}

Interactions with Healthcare Providers

The patients voiced that they need to communicate with healthcare providers online, which may address their individual needs and concerns.

G2: I downloaded the APP named "Haodaifu", which helps me to consult a famous rheumatologist. I conveyed my symptoms, then they give me some advice.

G7: Communicating with doctors is important. Some misleading information is from other patients with gout. But a lot of APPs cannot support that. I cannot receive a reply from the doctors.

G8: I want to sign a private doctor. He can give me suggestions for managing my condition.

Interactions with Fellow Patients

Patients received useful information regarding treatment and the experience of living with gout flares through interaction with fellow patients.

G4: Sometimes I can get some useful information when I talk with them (fellow patients). I tried taking medications which were recommended by a patient. He said it could prevent pain, he can also drink, and has not been suffering the pain for a long time.

G6: I think the communication with patients is helpful. Patients can share their experience living with gout. What medicine is more effective? How can we relieve and prevent the pain?

\section{Discussion}

This study investigated the experience of patients living with gout and identified their needs with respect to gout self-management support. Instrumental, psychological, and relational support emerged as themes describing their needs for gout self-management support, which could be utilized for developing a mobile APP.

\section{Embedding Diverse Health Education Information in the mHealth APP for Gout Self-Management}

Nine health education needs emerged from the qualitative data: basic disease-related knowledge; up-to-date medical news and information related to gout; dietary advice; lifestyle advice; associated comorbidities; prevention and management of an acute attack; treatment of tophi; ULT; and effects and side effects of medications for gout. All health education needs reflected the deficiency of patients in basic knowledge regarding gout, which is consistent with the findings of a previous study. ${ }^{22}$ Yin reported that $75.2 \%$ of the patients lack knowledge related to gout. ${ }^{23}$ Similarly, Li et $\mathrm{al}^{24}$ found that only $22.0 \%$ of the patients with gout had adequate diet-related knowledge. Patients also voiced that they did not receive adequate education on gout and disease-related information from healthcare providers. $^{25-27}$ Moreover, the present findings revealed that four of the 10 patients searched for gout-related information on the internet. Of note, the credibility and readability of the information were questioned. Jiménez-Liñán et $\mathrm{al}^{28}$ selected 85 websites to assess the content and readability of online resources of information related to gout. Most of them provided no/inaccurate information on the pathogenesis of gout, ULT, and its potential for cure; the information in $68.0 \%$ of these websites was rated at least fairly difficult. Johnston et $\mathrm{al}^{29}$ assessed 30 patient educational resources (18 printed, 12 websites) from six countries using the Flesch-Kincaid Grade Level score. Sixteen of the 30 resources received a score above Grade 9, which denotes highly complex information. Therefore, education on gout should be based on the needs of patients. Prior to posting on the internet, the accuracy and readability of these resources should also be verified by healthcare providers.

\section{The mHealth APP Should Provide Psychological Support to Patients with Gout}

Our findings suggest that patients with gout had a variety of negative emotions (eg, anxiety, depression, frustration) due to the pain, economic burden, fear of medicationrelated side effects, reduced social activities, change in 
employment, and disharmony of family relationships caused by gout. Howren et $\mathrm{al}^{30}$ conducted a systematic review to assess the prevalence of depression and anxiety among patients with gout; the pooled prevalence of depression and anxiety was $10.0 \%$ and $6.0 \%$, respectively. Gout was independently associated with a $42.0 \%$ higher risk of incident depression in older adults. ${ }^{31}$ The pain of gout attacks even led some patients to thoughts of suicide. $^{32} \mathrm{~A}$ cross-sectional study revealed that $26.0 \%$ of the patients experienced internalized stigma. ${ }^{33}$ Thus, healthcare providers should consider the mental health of patients and provide strategies to help them overcome negative emotions.

\section{The mHealth APP Should Assist in Promoting Health Behavior During the Process of Gout Self-Management}

The ability to set reminders, arranged appointments with a healthcare provider, and purchase medications through the mHealth APP could promote healthy behavior during the process of gout self-management. Lifelong management of gout is necessary, particularly the long-term usage of ULT. Adherence to ULT is the key to effective treatment of gout. However, the adherence rate to ULT is as low as $47.0 \%$ (95\% confidence interval: $42.0-52.0 \%){ }^{34}$ We found that forgetting to take medications contributed to this low adherence, which was also observed in a previous study. ${ }^{35}$ Setting reminders may be a solution to this problem. Guidelines for the management of gout ${ }^{5,6}$ recommend that the level of SUA should be monitored regularly to titrate the dosage of ULT, until the target for SUA is reached. Patients with gout should undergo periodic screening for associated comorbidities, which can help healthcare providers identify risk factors early. Setting reminders and hospital registration can help patients continue their interaction with healthcare providers in follow-up visits. Furthermore, the ability to purchase necessary products could help them self-medicate as soon as warning symptoms develop, which is considered an integral part in gout self-management.

\section{Limitations}

In this study, only male patients with gout in Sichuan were analyzed. However, there may be differences in preferences regarding health education and lifestyle adjustment according to the sex, region, and ethnicity. Female patients and those from different areas should be included in future studies. Such broader investigations could yield useful data for updating the aforementioned themes of patient needs.

\section{Conclusion}

This study investigated the experience of patients living with gout and identified their needs with respect to gout self-management support. The results showed that instrumental, psychological, and relational emerged as themes describing their needs for gout self-management support. The instrumental support included health education, hospital registration, setting reminders, and shopping. Psychological support referred to helping patients overcome various negative emotions. Relational support included interactions with doctors and fellow patients. The present findings indicate that healthcare providers or companies should develop mHealth APPs for gout selfmanagement based on the needs of patients.

\section{Abbreviations}

ULT, urate-lowering therapy; mHealth, mobile health; APPs, Applications; SUA, serum uric acid; BMI, body mass index.

\section{Acknowledgments}

The researchers are grateful to the patients who participated in this study for sharing their experiences regarding gout self-management and perception of mobile APPs.

\section{Funding}

This study was supported by the SCST (Science and Technology of Sichuan Province) (Grant No.: 2018FZ0110). The SCST was not involved in the stages from study design to submission of the paper for publication.

\section{Disclosure}

The authors report no conflicts of interest in this work.

\section{References}

1. The Lancet Rheumatology. Big little lies: challenging misperceptions of gout. Lancet Rheum. 2019;1(2):e75. doi:10.1016/S2665-9913(19) 30036-0.

2. Yu KH, Chen DY, Chen JH, et al. Management of gout and hyperuricemia: multidisciplinary consensus in Taiwan. Int $J$ Rheum Dis. 2018;21(4):772-787. doi:10.1111/1756-185X.13266

3. Kuo CF, Grainge MJ, See LC, et al. Epidemiology and management of gout in Taiwan: a nationwide population study. Arthritis Res Ther. 2015;17(1):13. doi:10.1186/s13075-015-0522-8 
4. Chen Y, Tang Z, Huang Z, et al. The prevalence of gout in mainland China from 2000 to 2016: a systematic review and meta-analysis. J Public Health. 2017;25:521-529. doi:10.1007/s10389-017-0812-5

5. Richette P, Doherty M, Pascual E, et al. 2016 updated EULAR evidence-based recommendations for the management of gout. Ann Rheum Dis. 2017;76(1):29-42. doi:10.1136/annrheumdis-2016-209707

6. Hui M, Carr A, Cameron S, et al. The british society for rheumatology guideline for the management of gout. Rheumatology. 2017;56 (7):e1-e20. doi:10.1093/rheumatology/kex156

7. Zhu Y, Pandya BJ, Choi HK. Comorbidities of gout and hyperuricemia in the US general population: NHANES 2007-2008. Am J Med. 2012;125(7):679-687. doi:10.1016/j.amjmed.2011.09.033

8. Zhang F. The Current Status and Influential Factors of SelfManagement in Gout Patients. Qingdao: Qingdao University; 2017.

9. Liang GY. Analysis of Follow-Up Status and Exploration of the IL-17 Expresssion Level for Patients with Gout. Beijing: Peking Union Medical College; 2016.

10. Scott IA, Scuffham P, Gupta D, Harch TM, Borchi J, Richards B. Going digital: a narrative overview of the effects, quality and utility of mobile apps in chronic disease self-management. Aust Health Rev. 2020;44(1):62-82. doi:10.1071/AH18064

11. Najm A, Gossec L, Weill C, Benoist D, Berenbaum F, Nikiphorou E. Mobile health apps for self-management of rheumatic and musculoskeletal diseases: systematic literature review. JMIR mHealth uHealth. 2019;7(11):e14730. doi:10.2196/14730

12. NEWZOO. 2020 global mobile market report. 2020. Available from: https://resources.newzoo.com/hubfs/Reports/2020_Free_ Global_Mobile_Market_Report.pdf?utm_campaign=Global\% 20 Mobile $\% 20$ Market\%20Report\%202020\&utm_medium= e mai $1 \&$ _ h s m i $=95373037 \&$ \& s e n c $=$ p 2 A N q t zMfoxwCCeNxDPb5sHydeesQX4GizTCG6JMzgWNbsCVJgjU4t1WiRb_fjG3dRACxuepc25xLfDAyjPtz8qHIrvh2Zyzw\&utm_con tent $=95373037 \& u$ tm_source $=$ hs_automation. Accessed May 9, 2021.

13. Li XC. Analysis on Possibility of Mobile Apps on Gout/ Hyperuricaemia: A New Management Model for Chronic NonInfectious Disease. Chengdu: Sichuan University; 2016.

14. Huang C, Zhan YY, Liu XY, et al. Review on the application of mobile health in nursing. Chin J Nur. 2019;54(8):1264-1269. doi:10.3761/j.issn.0254-1769.2019.08.031

15. Wang XR, Ding YX, Wang BQ. Application effect and enlightenment of mobile-health APP in continuity care. Chin Nur Res. 2019;33 (5):803-806. doi:10.12102/j.issn.1009-6493.2019.05.016

16. Xu XY, Wang JJ, Liu JP, Zhu PR, Luo WL. Research progress on application of mobile phone APP in health management. Chin EvidBased Nur. 2018;4(10):888-890. doi:10.12102/j.issn.2095-8668.20 18.10.006

17. Yin Y, Chen H. Letter to the editor: mobile applications for patients with gout in China. Clin Rheumatol. 2019;38(8):2281-2282. doi:10.1007/s10067-019-04622-1

18. Najm A, Nikiphorou E, Kostine M, et al. EULAR points to consider for the development, evaluation and implementation of mobile health applications aiding self-management in people living with rheumatic and musculoskeletal diseases. RMD Open. 2019;5(2):e001014. doi:10.1136/rmdopen-2019-001014
19. McCurdie T, Taneva S, Casselman M, et al. mHealth consumer apps: the case for user-centered design. Biomed Instrum Technol. 2012;46: (s2):49-56. doi:10.2345/0899-8205-46.s2.49

20. Neogi T, Jansen T, Dalbeth N, et al. 2015 Gout classification criteria: an American College of Rheumatology/European League Against Rheumatism collaborative initiative. Ann Rheum Dis. 2015;74 (10):1789-1798. doi:10.1136/annrheumdis-2015-208237

21. Colaizzi PF. Psychological research as the phenomenologist's view it. In: Existential Phenomenological Alternatives for Psychology. 1978:48-71.

22. Derksen C, Serlachius A, Petrie KJ, Dalbeth N. What say ye gout experts?" a content analysis of questions about gout posted on the social news website Reddit. BMC Musculoskelet Disord. 2017;18 (1):488. doi:10.1186/s12891-017-1856-y

23. Yin RL. A Survey of Treatment Adherence to Urate-Lowering Therapy in Chinese Gout Patients and Its Influencing Factors. Nantong: Nantong University; 2017.

24. Li ZX, Li QH, Mo YQ, Liu HJ, Zheng DH, Dai L. Questionnaire survey evaluating gout-related diet control Knowledge on patients with gout. J Sun Yat-Sen Uni(Med Sci). 2015;36(2):306-312.

25. Rai SK, Choi HK, Choi SHJ, Townsend AF, Shojania K, De Vera MA. Key barriers to gout care: a systematic review and thematic synthesis of qualitative studies. Rheumatology. 2018;57 (7):1282-1292. doi:10.1093/rheumatology/kex530

26. Panginikkod S, Hashim R, Ufion AJA, et al. Knowledge gaps in gout management: a cross sectional survey among internal medicine residents. J Gen Intern Med. 2018;33(s2):S246-S246.

27. Liddle J, Roddy E, Mallen CD, et al. Mapping patients' experiences from initial symptoms to gout diagnosis: a qualitative exploration. BMJ Open. 2015;5(9):e008323. doi:10.1136/bmjopen-2015-008323

28. Jimenez-Liñan LM, Edwards L, Abhishek A, Doherty M. Adequacy of online patient information resources on gout and potentially curative urate-lowering treatment. Arthritis Care Res (Hoboken). 2017;69 (5):748-752. doi:10.1002/acr.22981

29. Johnston ME, Treharne GJ, Chapman PT, Stamp LK. Patient information about gout: an international review of existing educational resources. J Rheumatol. 2015;42(6):975-978. doi:10.3899/jrheum.141442

30. Howren A, Zusman EZ, Rai SK, Shojania K, De Vera MA. Prevalence, incidence, determinants, and impacts of depression and anxiety in gout: a systematic review. Arthritis Rheumatol. 2018;70:s9.

31. Singh JA, Cleveland JD. Gout and the risk of incident depression in older adults. Psychiatry Res. 2018;270:842-844. doi:10.1016/j. psychres.2018.10.067

32. Morgan J. Gout is not a life sentence: be informed. Lancet Rheum. 2019;1(3):e144. doi:10.1016/S2665-9913(19)30074-8

33. Kleinstauber M, Wolf L, Jones ASK, Dalbeth N, Petrie KJ. Internalized and anticipated stigmatization in patients with gout. ACR Open Rheumatol. 2020;2(1):11-17. doi:10.1002/acr2.11095

34. Yin R, Li L, Zhang G, et al. Rate of adherence to urate-lowering therapy among patients with gout: a systematic review and meta-analysis. BMJ Open. 2018;8(4):e017542. doi:10.1136/bmjopen-2017-017542

35. Aung T, Myung G, FitzGerald JD. Treatment approaches and adherence to urate-lowering therapy for patients with gout. Patient Prefer Adherence. 2017;11:795-800. doi:10.2147/PPA.S97927 


\section{Publish your work in this journal}

Patient Preference and Adherence is an international, peer-reviewed, open access journal that focusing on the growing importance of patient preference and adherence throughout the therapeutic continuum. Patient satisfaction, acceptability, quality of life, compliance, persistence and their role in developing new therapeutic modalities and compounds to optimize clinical outcomes for existing disease states are major areas of interest for the journal. This journal has been accepted for indexing on PubMed Central. The manuscript management system is completely online and includes a very quick and fair peer-review system, which is all easy to use. Visit http:// www.dovepress.com/testimonials.php to read real quotes from published authors. 